\title{
The AGFA and AIRIS separators at ATLAS
}

\author{
B. B. Back $^{1}$ \\ ${ }^{1}$ Physics Division, Argonne National Laboratory, Argonne, IL 60439, USA
}

\begin{abstract}
The ATLAS facility is being augmented with two new separators in order to take full advantage of recent and future anticipated beam intensity upgrades to the accelerator. A novel concept is used for the Argonne Gas-Filled Analyzer (AGFA), which employs only two magnetic elements; a quadrupole singlet for vertical focusing and a multipole magnet that provides a dipole field for the separation and a quadrupole field for horizontal focusing of the reaction products. The design allows for placing Gammasphere or GRETINA at the target position. This arrangement enables studies of prompt gamma-ray emission from weakly populated trans-fermium nuclei and those near the doubly-magic $\mathrm{N}=\mathrm{Z}=50$ shell closures measured in coincidence with the recoils registered in the AGFA focal plane. The Argonne In-flight Radioactive Ion Separator (AIRIS) is a magnetic chicane that will be installed immediately downstream of the last ATLAS cryostat. It will be used to separate radioactive ion beams generated in flight by the primary beam at an upstream high-intensity production target. These beams will be further purified by a downstream RF sweeper and transported to a number of target stations including HELIOS, the Enge spectrograph, the FMA and Gammasphere.
\end{abstract}

\section{Introduction}

With the recent accelerator upgrades at ATLAS, which were achieved by replacing several cryostats with the original split-ring resonators with modern quarter-wave resonators, it is now possible to accelerate high-intensity beams of stable heavy ions. In anticipation of this development, we have undertaken a program of instrumentation upgrades in order to utilize these beams in an effective manner and to expand the research program at ATLAS. First, we have developed a novel design for a gasfilled separator, the Argonne Gas-Filled Analyzer, AGFA, chiefly to separate and study the structure of transuranic nuclei that can be produced in heavy-ion fusion reactions, but only with small cross sections. This endeavor requires both intense heavy-ion beams and efficient collection of the reaction products at the focal plane of the separator in order to characterize their decay properties.

Secondly, we have for two decades carried out a successful research program with light, radioactive beams produced in flight using various light charged-particle reactions, such as $(\mathrm{d}, \mathrm{p}),(\mathrm{d}, \mathrm{n}),(\mathrm{p}, \mathrm{n})$ etc., in inverse kinematics by focusing the primary, stable heavy-ion beam onto a production gas cell containing hydrogen or deuterium gas. The radioactive reaction products of interest are focused by a solenoid and subsequently separated from the primary beam by a magnetic bend downstream before being focused onto the target of the experimental setup. However, thus far this production facility is limited by being able to deliver radioactive beams only to the Enge spectrograph and HELIOS beam lines and by the fact that the gas cell can only tolerate primary beams of moderate intensity. Since an enhanced facility for producing in-flight radioac- tive beams will be able to support a wide range of experimental research, most in nuclear astrophysics and nuclear structure studies, we have designed a dedicated separator, the Atlas In-flight Radioactive Ion Separator, AIRIS, which will be installed immediately downstream from the last cryostat of the ATLAS linac such that all experimental stations in the facility can accept these beams and the full intensity of the primary beam can be utilized by providing effective shielding and increasing the distance between the primary beam dump and the experimental areas.

\section{AGFA}

The technique of collecting reaction products from fusion evaporation and separating them from the primary beam using the gas-filled separator technique [1] has proven very successful and it is now used almost exclusively in experiments aimed at strudying the structure of transactinide nuclei $[2,3]$ and synthesizing the heaviest elements [46]. We have developed a new, compact design [7] for such a separator with the dual functions of re-focusing the evaporation residues onto a limited area of the focal plane and separating them from the beam. With the AGFA design (see table 1), these functions are achieved by using only two magnetic components, 1) a vertically focusing quadrupole magnet, $\mathrm{Q}_{v}$, and 2) a dipole magnet, $\mathrm{D}_{m}$, that incorporates also a quadrupole component for horizontal focusing of the evaporation residues. This design concept, illustrated in Fig.1, has several attractive features, chiefly the possibility to place the Gammasphere array at the target position $\sim 84 \mathrm{~cm}$ upstream of the quadrupole magnet to allow for detection of promptly emitted $\gamma$ rays. In this 
Table 1. Some relevant parameters characterizing the AGFA separator. Note that the total flight-path length and the solid angle depends on the location of the target upstream of the quadrupole.

\begin{tabular}{|c|c|c|}
\hline Parameter & \multicolumn{2}{|c|}{ Value } \\
\hline Configuration & \multicolumn{2}{|c|}{$\mathrm{Q}_{v} \mathrm{D}_{m}$} \\
\hline Maximum bending power, $\mathrm{B} \rho$ & \multicolumn{2}{|c|}{ 2.5 Tesla-m } \\
\hline Maximum field at $\mathrm{Q}_{v}$ pole tip & \multicolumn{2}{|c|}{ 1.24 Tesla } \\
\hline Maximum field at $\mathrm{D}_{m}$ pole tip & \multicolumn{2}{|c|}{1.7 Tesla } \\
\hline Bend angle & \multicolumn{2}{|c|}{38 degrees } \\
\hline Target to $\mathrm{Q}_{v}$ distance & $40 \mathrm{~cm}$ & $84 \mathrm{~cm}$ \\
\hline Solid angle, $\Omega$ & $44 \mathrm{msr}$ & $22 \mathrm{~ms}$ \\
\hline Target to focal plane distance & $3.7 \mathrm{~m}$ & $4.3 \mathrm{~m}$ \\
\hline
\end{tabular}

position, the solid angle of the separator is about $22 \mathrm{msr}$. Alternatively, using a target position $40 \mathrm{~cm}$ upstream of the front of the quadrupole for experiments where prompt $\gamma$-ray detection is not needed, increases the solid angle to $44 \mathrm{msr}$. In typical experiments studying transuranic nuclei via heavy-ion fusion reactions, such as e.g. ${ }^{48} \mathrm{Ca}+{ }^{208} \mathrm{~Pb}$ $\rightarrow{ }^{254} \mathrm{No}+2 \mathrm{n}$, simulations show that there is a very good separation between the ${ }^{254}$ No recoils and the beam. In this case, $\sim 70 \%$ of the ${ }^{254}$ No recoils fall within an area of $64 \times 64 \mathrm{~mm}^{2}$, the size of a typical Double Sided Strip Detector, (DSSD). This relatively narrow focus allows for an efficient detection of subsequent $\gamma$ decays by using the Argonne X-array, which consists of five large HPGe Clover detectors that can be mounted around the focal plane chamber containing the $64 \times 64 \mathrm{~mm}^{2}$ DSSD implantation detector.

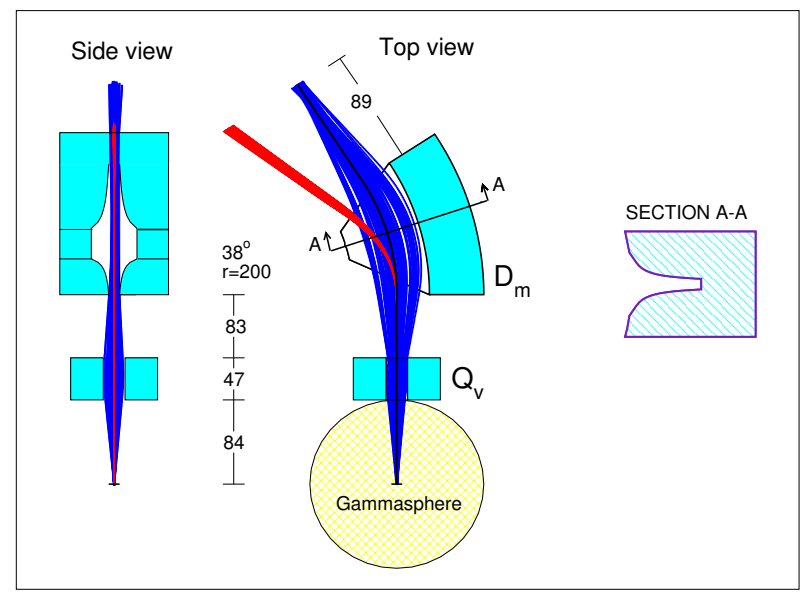

Figure 1. Schematic views of the AGFA separator with Gammasphere at the target position. The non-uniform character of the gap in the $\mathrm{D}_{m}$ magnet provides the horizontally focus. Dimensions are given in units of $\mathrm{cm}$.

An important feature of the design is the fact that the pole pieces of the $\mathrm{D}_{m}$ magnet constitute part of the vacuum envelope. The vacuum chamber inside the gap contains $\mathrm{O}-$ ring seals that mate with the pole surfaces. This vacuum chamber was fabricated by the magnet vendor, Buckley Systems [8], to ensure the effectiveness of this seal. Fig. 2 shows the two magnets mounted on the support stand in the final location at ATLAS and with the dipole chamber outfitted with two blank-off flanges in order to verify that good vacuum could be obtained also after re-assembly of the $\mathrm{D}_{m}$ magnet after shipment to Argonne.

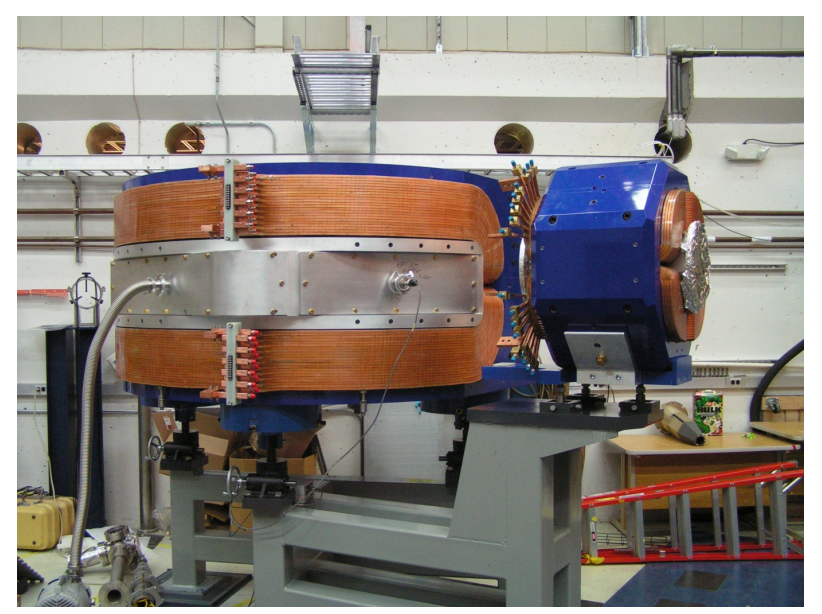

Figure 2. Photograph of the AGFA magnets at ATLAS taken during the vacuum test of the $\mathrm{D}_{m}$ vacuum chamber seal.

\subsection{Planned research program}

A very diverse research program is envisioned for the AGFA separator. It is anticipated that a main focus of this future research program will be in the study of the structure of the transuranic elements, which can be populated in heavy-ion fusion reactions involving beams of e.g. ${ }^{48} \mathrm{Ca}$, ${ }^{50} \mathrm{Ti}$, and ${ }^{54} \mathrm{Cr}$ impinging on targets around $\mathrm{Pb}$ and $\mathrm{Bi}$. The AGFA separator operated in conjunction with Gammasphere will provide a world-leading setup for this type of studies. In addition, it is anticipated that studies of exotic, heavy proton emitters will constitute a large part of the research program. A number of heavy proton emitters have been predicted in the At-Bk range of the most neutron-deficient nuclei. These have not yet been identified experimentally because of very low cross sections and possibly short half-lives. With the efficient collection of evaporation residues with AGFA, these studies can be pushed toward lower cross sections and the implementation of a digital readout system for the focal plane detector system and the short flight path of AGFA allows for more effective searches for weakly populated, short-lived proton emitters expected in this area. A long-standing area of interest in nuclear structure is the region around the doublymagic ${ }^{100} \mathrm{Sn}$ nucleus. Again, studies in this area have been hampered by the very small cross sections for populating the most neutron-deficient nuclei in this region, so the superior collection efficiency of AGFA coupled with the high $\gamma$-detection efficiency of Gammasphere offers hope that these studies can proceed at a more rapid pace. As always, a range of other possibilities may be realized once the capabilities of the AGFA-Gammasphere setup become better understood. Already, several new applications have been suggested. 


\subsection{Present status}

At present, April 2017, the AGFA separator is nearing completion. Several simple experimental tests are planned in order to understand and verify the expected capabilities of the instrument as part of the commissioning process. It is expected that this will be completed in early summer 2017 such that the experimental program can start immediately thereafter.

\section{AIRIS}

The possibility of producing a range of radioactive beams with masses up to about $\mathrm{A}=60$ removed by one to two nucleons from the stable nuclei is being realized by installing the AIRIS separator immediately downstream of the last cryostat of the ATLAS linac. Radioactive beams produced and separated from the primary beams and other contaminants can be transported to all downstream experimental stations. A schematic of the separator setup is given in Fig. 3. Some of the relevant design parameters are given in Table 2.

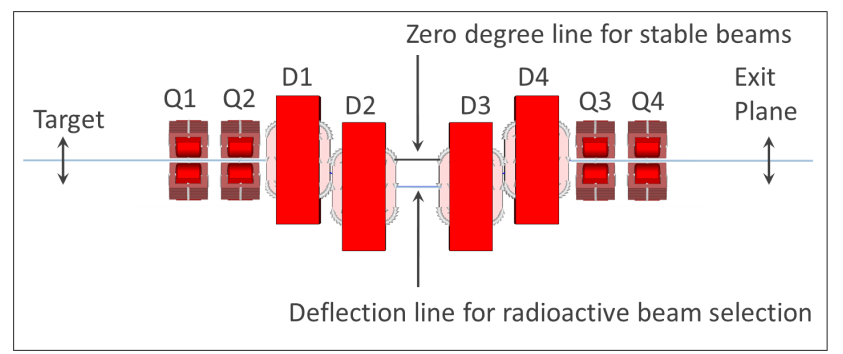

Figure 3. Schematic of the AIRIS separator consisting of four quadrupole singlets and four dipole magnets. The radioactive beam selection occurs at the mid-plane of the setup where the primary beam is stopped.

Radioactive beams produced by the interaction of the primary beams with the upstream high-intensity target are focused by a quadrupole doublet, Q1-Q2, to pass through a vertical slit opening at the mid-plane after having been bent about $25 \mathrm{~cm}$ down from the straight-through beam path. The primary beam will be stopped on either the upper or the lower slit depending on its magnetic rigidity relative to that of the selected radioactive beam. Just downstream of the slits, several diagnostics elements, such as a Faraday cup or a Si detector telescope, can be inserted into the radioactive beam path in order to ensure that the correct beam has been selected for further transport to the exit of the device.

Further downstream, the radioactive beam is brought back to the straight-through beam path by a second pair of dipole magnets and focused onto the exit plane by a second set of quadrupole magnets, Q3-Q4. At the exit plane, a second set of slits will further help to eliminate beam contaminants that managed to be transported up to this point. Here, again, various diagnostic elements can be used to characterize the beam particles that are allowed to proceed downstream. In cases where beam contaminants are not
Table 2. List of some relevant design parameters for the AIRIS separator.

\begin{tabular}{lc}
\hline \hline Parameter & Value \\
\hline Dipole bend angle & $22.5 \mathrm{deg}$ \\
Dipole radius & $1 \mathrm{~m}$ \\
Dipole full gap & $10 \mathrm{~cm}$ \\
Max. Dipole field & $1.75 \mathrm{Tesla}$ \\
Quadrupole full aperture & $15 \mathrm{~cm}$ \\
Max. quadrupole tip field & $1.0 \mathrm{Tesla}$ \\
Offset at mid-plane & $25 \mathrm{~cm}$ \\
Total length: Target to Exit slits & $7.1 \mathrm{~m}$ \\
Geometric acceptance & $75 \mathrm{mrad}$ \\
Energy dispersion at mid-plane & $1.25 \mathrm{~mm} / \%$ \\
\hline \hline
\end{tabular}

fully eliminated by the methods described above, a final clean-up may be necessary by using an existing RF (radiofrequency) sweeper. This device is currently installed in the Enge Spectrograph beam line, but will be re-located further upstream such that it can serve a larger number of experimental stations.

While the AIRIS separator is intended for selecting and transporting secondary, in-flight beams, it is important that it also allows for the transport of stable beams directly from the ECR source or radioactive beams of ${ }^{252} \mathrm{Cf}$ fission fragments from the CARIBU source directly to the experimental stations as such beams are used in the majority of experiments performed at ATLAS. This is achieved by de-energizing the four dipole magnets in AIRIS and using only the two quadrupole doublets Q1-Q2 and Q3-Q4 for focusing of these beams. The upper slits at the mid-plane are, therefore, fully retractable to allow for the transport of straight-through beams.

The selectivity and efficiency of this design [9] has been characterized by a large number of transport simulations using the TRACK beam transport code [10] in order to arrive at an optimal design solution that can be realized within the spatial constraints of the ATLAS tunnel.

In the particle transport simulations, we concentrated on following: the trajectories of the in-flight beam, the primary beam including its small angle scattering in the production target (beam core), the elastically scattered beam particles (beam tail) as well as possile contaminants produced in he reaction. As an example, Fig. 4 illustrates the separation of a ${ }^{56} \mathrm{Ni}$ beam produced in a two-proton adding reaction, namely ${ }^{54} \mathrm{Fe}\left({ }^{12} \mathrm{C},{ }^{10} \mathrm{Be}\right)$, using a $2 \mathrm{mg} / \mathrm{cm}^{2}$ production target. At the mid-plane focus there is good separation between the primary beam core and the in-flight ${ }^{56} \mathrm{Ni}$ beam in the $28^{+}$charge state, but the ${ }^{55} \mathrm{Co}^{27+}$ contaminant, produced in the one-proton adding reaction, clearly extends into the acceptance region of the $y=-5 \mathrm{~cm}$ to $5 \mathrm{~cm}$ opening of the mid-plane slits. However, this contaminant follows trajectories that end up above the in-flight beam at the exit focal plane, where they are easily removed by a set of vertical slits located at this position.

In order to characterize the capabilities of AIRIS, we have performed a large number of TRACK simulations for representative in-flight beams in the mass range up to $\mathrm{A} \sim 60$. The assumptions concerning the angle-integrated 


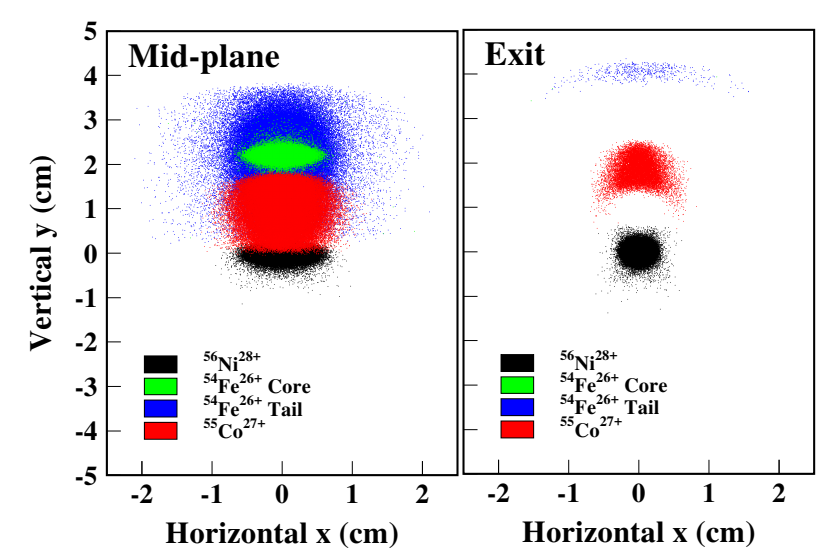

Figure 4. The left panel shows the transverse distribution of secondary ${ }^{56} \mathrm{Ni}^{28+}$ ions at the AIRIS mid-plane (black dots) along with those of the ${ }^{54} \mathrm{Fe}^{26+}$ beam core (green dots) and the ${ }^{54} \mathrm{Fe}^{26+}$ beam tail (blue dots). A secondary beam contaminant, ${ }^{55} \mathrm{Co}^{27+}$ (red dots), is also transported to the AIRIS mid-plane, but this component is not completely removed by the mid-plane slits which in this simulation select ions within the range $-5 \mathrm{~mm}<$ $\mathrm{y}<5 \mathrm{~mm}$. The right panel shows the transverse distribution at the exit (focal plane) of particles that have passed through the mid-plane slits.

production cross section, $\sigma_{p r}$, the charge state fraction, $F(q)$, and the primary beam energy are listed in Table 3 along with the resulting secondary beam energy, the transmission, $T$, through AIRIS to the exit plane, and the resulting in-flight beam intensity. These simulations are all based on an effective target thickness of $2 \mathrm{mg} / \mathrm{cm}^{2}$ and a primary beam intensity of $1 \mathrm{p} \mu \mathrm{A}$. Further details on these simulations and the relevant input parameters are given in Ref. [11]

\subsection{High-intensity production target}

Several options exist for the design of a production target that can tolerate heavy-ion beam intensities in the range of $1 \mathrm{p} \mu \mathrm{A}$. For solid target materials, such as $\mathrm{C}$ or Be, rotating target wheels are well proven to be able to accomodate beam intensities of this magnitude. However, concerning gaseous targets such as deuterium, hydrogen, and helium, it is not possible to employ the present method with gas cells because of the fragility of the entrance and exit windows. Instead, we have developed a high intensity target that generates a thin, liquid film by impinging two oil jets of $\sim 0.5 \mathrm{~mm}$ diameter on each other inside a vacuum chamber. This mechanism generates a thin oil film of areal thickness $\sim 1-2 \mathrm{mg} / \mathrm{cm}^{2}$ needed for the primary target. The low vapor pressure of regular pump oil ensures that high vacuum can be maintained in the beam line. Several baffles and an in-line cold trap further ensure that the oil does not migrate along the beamline. The hydrogen content of the oil makes this target ideal for proton-induced reactions, e.g. $(\mathrm{p}, \mathrm{n})$ and $(\mathrm{p}, \mathrm{d})$. However, in order to use deuteron initiated reactions, such as $(\mathrm{d}, \mathrm{n})$ and $(\mathrm{d}, \mathrm{p})$, a deuterated pump oil is required, an option that we're presently researching.
In addition, it is possible that the liquid film target can also be used in carbon induced reactions.

\subsection{Planned research program}

One of the research areas that will benefit from the development of the AIRIS separator is nuclear astrophysics. Over the last two decades, such studies have been carried out with great success using in-flight beams with a gas-cell production target, a superconducting collector solenoid and a single magnetic bend in the beam line leading to either the Enge Splitpole Spectrograph or HELIOS. With the implementation of AIRIS, higher beam intensities, heavier and more exotic beams can be provided for such studies. In addition, these beams are expected to enable a strong research program in structure studies of exotic nuclei by using the HELIOS spectrometer for studies of transfer reactions or inelastic scattering. Nuclear structure studies based on $\gamma$-ray detection using either Gammasphere of GRETINA will also benefit from the in-flight beams that AIRIS wil be able to provide.

\subsection{Status}

The implementation of the AIRIS separator is presently at the following stage. All the main components are under contract with outside companies with expected delivery to Argonne in the early summer of 2017. The installation into the ATLAS tunnel is, however, very disruptive to the ongoing research program and detailed pre-planning and scheduling of this phase is of paramount importance. This phase is, thus, scheduled to coincide with the annual month-long accelerator maintenance period that includes the last week of December 2017 and January 2018. However, we anticipate that this down time has to be extended into February or early March 2018 before the beam transport through the ATLAS tunnel can be resumed. The commissioning of AIRIS will then follow and the first radioactive beams from this facility can be used in research in Spring 2018.

\section{Summary}

Two new devices that will substantially enhance the research capabilities of the ATLAS facility have been presented. The AGFA gas-filled separator is presently under construction and will support a wide-ranging research program in studies of trans-uranium nuclei, exotic proton emitters and very neutron deficient nuclides near the ${ }^{100} \mathrm{Sn}$ doubly-closed shell. The combination of this device with high-efficiency $\gamma$ detector arrays, such as Gammasphere and GRETINA, holds promise for extending nuclear structure studies into exotic regions of the nuclear chart that were previously inaccessible because of small cross sections for producing the nuclei of interest. It is clear, however, that the general property of very high collection efficiency of this device will also inspire research in areas that have not yet been foreseen. The AIRIS separator is being implemented in order to substantially enhance the current capabilities for in-flight production of 
Table 3. List of in-flight radioactive beams, reactions used to produce them, primary and secondary beam energies, assumed angle-integrated cross sections, $\sigma_{p r}$, radioactive beam transmission to the AIRIS exit plane, $T$, the percentage of the charge state used,

$F(q)$, and secondary beam intensities for 19 representative reactions using a $2 \mathrm{mg} / \mathrm{cm}^{2}$ target and $1 \mathrm{p} \mu \mathrm{A}$ primary beam.

\begin{tabular}{|c|c|c|c|c|c|c|}
\hline $\begin{array}{l}\text { Second. } \\
\text { Beam }\end{array}$ & Reaction & $\begin{array}{c}\text { Sec.[Pri.] } \\
\text { Energy } \\
(\mathrm{MeV} / \mathrm{u})\end{array}$ & $(\mathrm{mb})$ & $(\%)$ & $F(q)$ & $\begin{array}{c}\text { Rate } \\
\text { Exit } \\
\text { (ions/s) }\end{array}$ \\
\hline${ }^{11} \mathrm{Li}^{3+}$ & ${ }^{14} \mathrm{C}\left({ }^{50} \mathrm{Ti},{ }^{53} \mathrm{Mn}\right)$ & $7.6[9]$ & 0.01 & 50 & 100 & $4.0 \times 10^{3}$ \\
\hline${ }^{12} \mathrm{Be}^{4+}$ & ${ }^{14} \mathrm{C}\left({ }^{26} \mathrm{Mg},{ }^{28} \mathrm{Si}\right)$ & 15.9 [15] & 0.2 & 50 & 100 & $8.0 \times 10^{4}$ \\
\hline${ }^{15} \mathrm{C}^{6+}$ & ${ }^{14} \mathrm{C}(\mathrm{d}, \mathrm{p})$ & $4.1[5]$ & 50 & 60 & 100 & $2.4 \times 10^{7}$ \\
\hline${ }^{16} \mathrm{C}^{6+}$ & ${ }^{14} \mathrm{C}\left({ }^{26} \mathrm{Mg},{ }^{24} \mathrm{Mg}\right)$ & $12.0[15]$ & 1 & 80 & 100 & $6.4 \times 10^{5}$ \\
\hline${ }^{16} \mathrm{C}^{6+}$ & ${ }^{18} \mathrm{O}\left({ }^{9} \mathrm{Be},{ }^{11} \mathrm{C}\right)$ & $10.4[10]$ & 0.2 & 50 & 100 & $8.0 \times 10^{4}$ \\
\hline${ }^{14} \mathrm{O}^{8+}$ & ${ }^{14} \mathrm{~N}(\mathrm{p}, \mathrm{n})$ & $14.0[15]$ & 30 & 50 & 100 & $1.2 \times 10^{7}$ \\
\hline${ }^{19} \mathrm{O}^{8+}$ & ${ }^{18} \mathrm{O}(\mathrm{d}, \mathrm{p})$ & $14.1[15]$ & 50 & 60 & 100 & $2.4 \times 10^{7}$ \\
\hline${ }^{18} \mathrm{Ne}^{10+}$ & ${ }^{16} \mathrm{O}\left({ }^{12} \mathrm{C},{ }^{10} \mathrm{Be}\right)$ & $7.1[10]$ & 1 & 40 & 100 & $3.2 \times 10^{5}$ \\
\hline${ }^{25} \mathrm{Al}^{13+}$ & ${ }^{24} \mathrm{Mg}(\mathrm{d}, \mathrm{n})$ & $3.8[5]$ & 50 & 80 & 42 & $1.3 \times 10^{7}$ \\
\hline${ }^{26} \mathrm{Al}^{13+}$ & ${ }^{26} \mathrm{Mg}(\mathrm{p}, \mathrm{n})$ & $9.0[10]$ & 50 & 80 & 86 & $2.8 \times 10^{7}$ \\
\hline${ }^{34} \mathrm{Si}^{14+}$ & ${ }^{36} \mathrm{~S}\left({ }^{9} \mathrm{Be},{ }^{11} \mathrm{C}\right)$ & $9.7[10]$ & 0.2 & 50 & 81 & $6.5 \times 10^{4}$ \\
\hline${ }^{37} S^{16+}$ & ${ }^{36} \mathrm{~S}(\mathrm{~d}, \mathrm{p})$ & $8.9[10]$ & 50 & 40 & 72 & $1.1 \times 10^{7}$ \\
\hline${ }^{33} \mathrm{Cl}^{17+}$ & ${ }^{32} \mathrm{~S}(\mathrm{~d}, \mathrm{n})$ & $9.1[10]$ & 50 & 80 & 67 & $2.1 \times 10^{7}$ \\
\hline${ }^{38} \mathrm{Ca}^{20+}$ & ${ }^{36} \mathrm{Ar}\left({ }^{12} \mathrm{C},{ }^{10} \mathrm{Be}\right)$ & $8.2[10]$ & 1 & 80 & 52 & $3.3 \times 10^{5}$ \\
\hline${ }^{49} \mathrm{Ca}^{20+}$ & ${ }^{48} \mathrm{Ca}(\mathrm{d}, \mathrm{p})$ & $9.2[10]$ & 50 & 25 & 52 & $5.2 \times 10^{6}$ \\
\hline${ }^{50} \mathrm{Ca}^{20+}$ & ${ }^{48} \mathrm{Ca}\left({ }^{18} \mathrm{O},{ }^{16} \mathrm{O}\right)$ & $9.1[10]$ & 1 & 80 & 52 & $3.4 \times 10^{5}$ \\
\hline${ }^{41} \mathrm{Sc}^{21+}$ & ${ }^{40} \mathrm{Ca}(\mathrm{d}, \mathrm{n})$ & $8.7[10]$ & 50 & 80 & 48 & $1.5 \times 10^{7}$ \\
\hline${ }^{56} \mathrm{Ni}^{27+}$ & ${ }^{54} \mathrm{Fe}\left({ }^{12} \mathrm{C},{ }^{10} \mathrm{Be}\right)$ & $8.6[10]$ & 1 & 80 & 44 & $2.8 \times 10^{5}$ \\
\hline${ }^{56} \mathrm{Ni}^{28+}$ & ${ }^{54} \mathrm{Fe}\left({ }^{12} \mathrm{C},{ }^{10} \mathrm{Be}\right)$ & $8.6[10]$ & 1 & 80 & 14 & $9.0 \times 10^{4}$ \\
\hline
\end{tabular}

radioactive beams close to the line of stability. This development builds on our experience of producing such beams over the past two decades using a relatively simple setup, which has still proven very productive in terms of physics results. Using high-intensity beams from the upgraded ATLAS linac and the dedicated AIRIS separator higher intensity in-flight beams can be produced and used in a broad research program.

The AFGA collaboration includes: B.B. Back, W.F. Henning, R.V.F. Janssens, T.L. Khoo, J.A. Nolen , D.H. Potterveld , J. Rohrer, G. Savard, and D. Seweryniak, Argonne National Laboratory, M. Paul, Hebrew University, Jerusalem, P. Chowdhury and C.J. Lister, University of Massachusetts - Lowell, W.B. Walters, University of Maryland, P.J. Woods, University of Edinburgh, K. Gregorich, Lawrence Berkeley National Laboratory, and W. Loveland, Oregon State University.

The AIRIS collaboration consists of: M. Alcorta (now at Triumf, Canada), B. B. Back, C. Dickerson, C. R. Hoffman, B. P. Kay, A. Levand, S. Manikonda (now at AML, Florida), B. Micklich, B. Mustapha, J. Nolen, P. Ostroumov (now at Michigan State University), R. C. Pardo, K. E. Rehm, G. Savard, J. P. Schiffer and D. Seweryniak, Argonne National Laboratory.

This work was supported by the U.S. Department of Energy, Office of Nuclear Physics, under Contract No. DE-AC02-06CH11357 and uses resources from ATLAS, an Office of Science user facility.

\section{References}

[1] M. Paul et al., Nucl. Inst. Meth. A 277, 418 (1989)

[2] M. Leino et al., Nucl. Inst. Meth. B 99, 653 (1995)

[3] V. Ninov and K.E. Gregorich, in ENAM 98, Exotic Nuclei and Atomic Masses, edited by B. M. Sherrill, D. J. Morrissey, and C. N. Davids, AIP Conf. Proc. No. 455 AIP, Woodbury, 1998, p. 704

[4] K. Subotic, Yu. Ts. Oganessian, V. K. Utyonkov, Yu. V. Lobanov, F. Sh. Abdullin, A. N. Polyakov, Yu. S. Tsyganov, and O. V. Ivanov, Nucl. Instrum. Methods Phys. Res. Sec. A 481, 71 (2002)

[5] A. Semchenkov et al. Nucl. Instr. Meth. B, 266, 4153 (2008)

[6] K. Morita et al., Nucl. Inst. Meth. B 70, 220 (1992)

[7] The AGFA design and associated simulations are due to David Potterveld, Argonne National Laboratory.

[8] Buckley Systems, Mt Wellington, Auckland, New Zealand, http://www.buckleysystems.com/

[9] The AIRIS design and associated ion-beam simulations are due to Brahim Mustapha, Argonne National Laboratory.

[10] V.N. Aseev, P.N. Ostroumov, E.S. Lessner and B. Mustapha in Proc. 2005 Part. Accel. Conf., Knoxville, TN, TPAT029, p. 2053 (2005)

[11] http://www.phy.anl.gov/airis/index.html 